\title{
Course of perinatal depressive symptoms among South African women: associations with child outcomes at 18 and 36 months
}

\author{
Emily Claire Garman ${ }^{1}(1) \cdot$ Annibale Cois $^{2,3} \cdot$ Mark Tomlinson $^{4} \cdot$ Mary Jane Rotheram-Borus ${ }^{5} \cdot$ Crick Lund $^{1,6}$
}

Received: 26 September 2018 / Accepted: 21 January 2019 / Published online: 25 February 2019

(c) The Author(s) 2019

\begin{abstract}
Purpose Latent modelling was used to identify trajectories of depressive symptoms among low-income perinatal women in South Africa. Predictors of trajectories and the association of trajectories with child outcomes were assessed.

Methods This is a secondary analysis of data collected among women living in Cape Town settlements $(N=446)$. Participants were eligible if pregnant and 18 years or older, and included in the analysis if allocated to the control arm (routine perinatal care). Participants were excluded in case of non-singleton birth and baby death. Follow-up assessments were at 2 weeks, 6-, 18-, and 36-month postpartum. Trajectories of depressive symptoms were based on the Edinburgh Postnatal Depression Scale scores until 18-month postpartum, using latent class growth analysis. Child physical, cognitive, socioemotional, and behavioural outcomes were assessed at 18 and/or 36 months. Univariate and multivariate regressions were used to identify predictors of trajectories and differences in child outcomes.

Results Four trajectories were identified: chronic low (71.1\%), late postpartum (10.1\%), early postpartum (14.4\%), and chronic high (4.5\%). Low social support, unwanted pregnancy, and risky drinking were associated with the chronic high trajectory; unemployment and HIV-positive status with the early postpartum trajectory; and intimate partner violence with the late postpartum trajectory. Weight-to-length and weight-for-age $z$-scores at 18 months, and weight-for-age $z$-scores, length-for-age $z$-scores, emotional symptom, and peer problem scores at 36 months differed across trajectories.

Conclusions Severe depressive symptoms in postpartum period have a lasting effect on child physical and socio-emotional outcomes. Multiple screening throughout pregnancy and 1-year postpartum is essential.
\end{abstract}

Keywords Latent class growth analysis $\cdot$ Trajectories $\cdot$ Depression $\cdot$ Perinatal $\cdot$ Child development

Joint senior authors: Mary Jane Rotheram-Borus and Crick Lund.

Electronic supplementary material The online version of this article (https://doi.org/10.1007/s00127-019-01665-2) contains supplementary material, which is available to authorized users.

Emily Claire Garman

emily.garman@uct.ac.za

1 Department of Psychiatry and Mental Health, Alan J Flisher Centre for Public Mental Health, University of Cape Town, Building B, 46 Sawkins Road, Rondebosch, Cape Town, Western Cape 7700, South Africa

2 Division of Epidemiology and Biostatistics, School of Public Health and Family Medicine, University of Cape Town, Cape Town, Western Cape, South Africa

3 Burden of Disease Research Unit, South African Medical Research Council, Francie van Zijl Drive, Parow Valley, PO Box 19070, Tygerberg 7505, South Africa

\section{Introduction}

Perinatal depression, broadly defined by the World Health Organization (WHO) as major depression occurring during pregnancy and the first postpartum year, is experienced by

4 Department of Psychology, Institute for Child and Adolescent Health Research, Stellenbosch University, Maitland, Private Bag X1, Stellenbosch, Western Cape 7602, South Africa

5 University of California, UCLA Psychiatry and Behavioral Sciences, 10920 Wilshire Blvd, Suite 350, Box 957051, Los Angeles, CA 90024, USA

6 Population Research Department, Centre for Global Mental Health, Health Service, Institute of Psychiatry, Psychology and Neuroscience, King's College London, London, UK 
$13 \%$ of women living in low- and middle-income countries (LMICs) [1]. Prevalence in South Africa has consistently been greater, with antenatal depressed mood experienced by $21-39 \%$ of women antenatally [2-10], and by $24-35 \%$ of mothers postnatally. Extensive research has been conducted to document the effects of perinatal depression on children's health and development, most of which stems from high-income countries (HICs) and concentrates on socio-emotional and cognitive development [11-14].

In LMICs, evidence has instead largely focused on physical outcomes, such as child physical growth, which is a key indicator of children's health and nutritional status [15]. This is reasonable, given that, in LMICs, poor growth, malnutrition, and infections are the leading causes of under-five mortality $[16,17]$. The majority of evidence comes from South Asia, however, and remains mixed in Sub-Saharan Africa $[18,19]$. There is emerging evidence on the impact of perinatal depression on emotional and behavioural development in LMICs [18]. In a South African birth cohort study, there was an association between high maternal depressive symptoms at 6-month postpartum and greater externalising problems among 2-yearold children [20]. However, no associations were found between severe antenatal depressive symptoms and social withdrawal at 10-12-month postpartum among HIVinfected mothers and infants in Cape Town [5]. Finally, an association was found between postnatal depression at 2 months and insecure infant attachment at 18-month postpartum in a study conducted in a peri-urban settlement near Cape Town [21].

Most studies are cross sectional, however, or include only one assessment of depressive symptoms, thus ignoring the complex and episodic nature of the disorder or the possibility that symptoms are a continuation of prepregnancy depression $[22,23]$. Such methods have led researchers to ignore the importance of symptom chronicity $[14,25]$, and instead identify 'sensitive' periods, during which depressive symptoms are thought to have especially detrimental effects on child development [13, 24].

Emerging evidence from longitudinal studies in HICs and LMICs indicates that chronicity of depression is more important in predicting poorer child outcomes than are timing or severity of depression [25, 26]. For example, Rotheram-Fuller et al. (2018)'s study in South Africa indicated that there is a greater risk of stunting, and increased internalising and externalising problems among children of mothers with chronic symptoms, but not among mothers, whose symptoms were only present during pregnancy or only postnatally [27]. Chronicity is usually measured as the number of times women screen positive on a depression instrument over the course of a study. Because severe episodes are more likely to last longer, it has been argued that this way of conceptualising chronicity is flawed, as it confounds severity with chronicity [28, 29]. In addition, it relies on an arbitrary cutoff for risk of depression, which can vary by population and measurement tool used.

Studies which have used more complex longitudinal analyses, such as growth curve mixture modelling (GCMM), have enabled the identification of latent subgroups of women with both chronic and transient symptom trajectories during the perinatal period, in both HICs [30] and LMICs [31, 32]. This allowed investigators to disentangle severity from chronicity and moving away from the dichotomisation of depressive symptoms. Several studies, mostly from HICs, have also made use of this modelling technique to compare child outcomes across different trajectories [33]. Overall, studies indicated that children of mothers experiencing severe or subclinical chronic depressive symptoms were more likely to have developmental problems, compared to children of mothers with severe, but transient, symptoms.

More research is needed in LMICs, where the course of perinatal depressive symptoms and the patterns of risk and resilience among children of mothers with perinatal depression may differ from HICs, given the contexts of food insecurity, HIV, violence, and increased risk of illness among children [14, 15, 34]. The aim of the present study was, therefore, to address this gap. With the use of a person-centred latent approach to identify different depressive symptom trajectories among low-income women in South Africa, this study's objectives were first to identify predictors of different trajectories, and second, to assess whether children of mothers with different trajectories showed different physical, cognitive, socio-emotional, and behavioural outcomes at 18 and 36 months of age.

\section{Methods}

\section{Design}

This study is a secondary analysis of data collected as part of a cluster randomised controlled trial (RCT) among pregnant women living in peri-urban settlements in Cape Town, South Africa [35]. The aim of the cluster RCT was to assess the effect of a perinatal counselling intervention on a range of maternal and child health and nutrition outcomes. In already published work, the intervention did not have any effect on the participants' depressive symptoms [36, 37]. However, some differences in child physical outcomes and mothers' health behaviour were found between the two arms among depressed participants $[36,38,39]$. Therefore, only participants in the control arm were considered in this analysis. Data collection methods were described previously [35], but are briefly summarised here. 


\section{Setting and randomisation}

Forty neighbourhoods among three peri-urban settlements were selected as recruitment areas. With the use of aerial maps, street intercept surveys and street observation, 26 neighbourhoods were paired based on several characteristics, such as number of households, distance to an antenatal clinic, type of housing, and sanitation. The remaining neighbourhoods were excluded due to variability on these criteria. Neighbourhoods in matched pairs were randomised into the control or intervention arm using simple randomisation. Of the 13 matched pairs of neighbourhoods, 1 pair was excluded after 6 months due to too few pregnancies being reported (Online Resource 1).

\section{Recruitment and participants}

Pregnant women who were 18 years or older were identified by recruiters, local township women familiar with the neighbourhood's residents, who conducted house-to-house visits and obtained consent from eligible participants to be contacted again. The research team then obtained informed consent and recruited participants from an assessment centre situated locally. Recruitment took place between May 2009 and September 2010.

Due to slow recruitment, late-entry control participants were recruited after giving birth [35]; these participants did not have antenatal data and so were excluded from the analysis. Participants who gave birth to twins, died or whose baby died during the course of the study were also excluded.

\section{Procedure}

Participants in the control arm did not receive any intervention, besides the standard antenatal and postnatal care provided in the antenatal and well-baby clinics in Cape Town. Assessments were conducted by local women from the community, recruited for this study, trained, certified, and supervised weekly by a clinical psychologist. All data were collected using android devices linked to an online data collection programme. Assessments were conducted in English or isiXhosa, depending on participants' preferences. Participants were followed-up four times after the baseline assessment: approximately 2 weeks after birth, and at 6-, 18-, and 36-month postpartum. At 18-month postpartum, $8.5 \%$ of participants $(n=38)$ were lost to follow-up; this doubled at 36-month postpartum $(n=73 ; 16.4 \%)$.

\section{Measures}

Assessments were translated and back translated into isiXhosa, as per standard guidelines [40], and included a range of maternal and child measures [35]. These are briefly described below.

\section{Demographic and obstetric characteristics}

Self-reported demographic information collected at baseline included age, education, marital, and employment status. Multiple correspondence analysis was employed to create a binary asset-based index of wealth (lower or higher wealth) to use as a proxy for socio-economic status at baseline [41], based on economic and asset-related measures. Self-reported obstetric measures, such as gestation at recruitment, gravidity, parity, whether the pregnancy was planned or wanted, and previous miscarriages were also collected, and so was HIV status.

\section{Social characteristics}

The number of friends and relatives the participants had, as well as the frequency of contact, were recorded as a measure of social support at baseline. Whether the participant was living with the father of the baby was also recorded. Finally, participants reporting having been slapped, shoved, punched or threatened with a weapon by their partner in the past 12 months were classified as experiencing physical intimate partner violence (IPV).

\section{Health}

Depressive symptoms were assessed using the Edinburgh Postnatal Depression Scale (EPDS; [42]), a ten-item Likert scale instrument assessing symptoms in the past week. Scores range from 0 to 30, with a greater score suggesting more severe depressive symptoms. This instrument has been validated among antenatal and postnatal women [43], and among isiXhosa-speaking women [44, 45]. Internal reliability of the EPDS was good at baseline (Cronbach's $\alpha=0.88$ ).

Alcohol consumption during pregnancy (after discovery) was assessed using the Derived Alcohol Use Disorder Identification Test (AUDIT-C; [46])—a three-item version of the original ten-item AUDIT [47]. The last item related to the number of times three or more drinks was consumed in one sitting in the past month to reflect the definition of risky drinking for women in South Africa [48]. Scores range from 0 to 12 , and a score of three or more is indicative of risky drinking [46]. The AUDIT-C correlates well with the AUDIT [46], and has been used in previous research among Cape Town's informal settlements [49]. Internal consistency at baseline was good $(\alpha=0.86)$. 


\section{Child outcomes}

Physical outcomes were assessed at 18- and 36-month postpartum, by measuring the infant's length and weight, using scales that were calibrated weekly. These were transformed into $z$-scores based on age- and gender-adjusted norms [50].

The motor and cognitive subscales of the Bayley Scales of Infant Toddler Development [51] were administered at 18 months to assess infants' cognitive and behavioural outcomes. Scaled scores, adjusted for age, were calculated for each subscale. Children's executive functioning, including working memory and attention shifting, was assessed at 36-month postpartum using the non-verbal Executive Functioning Battery [52]. This scale consists of three sections: silly sounds (36 questions), something's the same (28 questions), and operation span (16 questions). Scores for each section were generated by summing all correct responses.

Participants completed two instruments to assess children's socio-emotional and behavioural outcomes at 36 months: the Child Behaviour Checklist (CBCL; [53]) and the Strength and Difficulty Questionnaire (SDQ; [54]). The CBCL is a 99-item 3-point Likert scale questionnaire assessing children's externalising, internalising, and inattention-hyperactivity problems. The SDQ comprises 25 items measuring conduct and emotional symptoms, hyperactivity and peer relationships, all of which fall under 'total difficulties', as well as prosocial behaviour. The SDQ has been translated and used in a previous research in Cape Town among older children and adolescents [55].

\section{Statistical analyses}

Non-parametric tests were used to compare baseline sociodemographic, obstetric, and health characteristics between participants who were included and excluded from the analyses, and between participants with and without data at 18and 36-month postpartum (Chi-square and Mann-Whitney $U$ tests, as appropriate). The analysis was then conducted in two steps-first a latent class growth analysis (LCGA), a type of GCMM, in Mplus version 8.0 [56] and a series of multivariate analyses in Stata version 14 [57].

\section{Identification of classes}

With the use of LCGA, EPDS scores from baseline to 18-month postpartum were used to identify latent groups of individuals with similar growth curves (classes) over the extended postpartum period. To reflect the non-equidistant times between assessments [58], factor loadings were set to 0 (baseline), 0.35 (2-week postpartum), 0.90 (6-month postpartum), and 2.10 (18-month postpartum). Participants completed on average 3.9 assessments (SD 0.50 ) from baseline to 18 -month postpartum. Missing data were assumed to be missing at random and were dealt with full information maximum likelihood estimation within Mplus. A pseudo-maximum likelihood approach (Huber-White sandwich estimator [59]) was implemented to adjust the estimates' standard errors for clustering by neighbourhood.

In a preliminary single-class analysis, the quadratic function fits the data significantly better that a linear one [60]. A quadratic polynomial growth function was thus chosen to allow for non-linear trajectories in all subsequent models. The optimal number of classes for the final model was chosen by comparing models with different numbers of classes according to the Bayesian and Akaike Information Criteria [61, 62], the entropy [63], and the average probability of class membership (posterior probability). The Lo-Mendel-Rubin test (LMRT) [64] and Bootstrap Likelihood Ratio Test (BLRT) [65] were also used to test the difference in fit between successive models. Finally, the size and theoretical interpretability of classes were also considered. After fitting the final model, participants were assigned to latent classes based on their highest posterior probability.

\section{Predictors and outcomes of trajectories}

To identify predictors of trajectories generated through the LCGA, a series of univariate unadjusted multinomial logistic regressions were conducted, with class membership as outcome, and baseline socio-demographic, obstetric and health characteristics as predictors. Class membership was then entered as a predictor in linear and logistic regressions to assess continuous and binary child outcomes, respectively, at 18 and 36 months. Each of these models were adjusted for age, education and wealth status, factors shown to be confounders in the relationship between maternal depression and child outcomes [15, 63-68]. To avoid reporting bias due to concurrent depressive symptoms, known to affect maternal reports of child behaviour [11, 69], models assessing maternal reports of child outcomes at 36 months were adjusted for participants' EPDS score. This was not necessary for child outcomes at 18 months, however, since the EPDS was used to generate the latent trajectories of depressive symptoms.

\section{Ethics}

The cluster RCT was approved by the Institutional Review Boards of the University of California Los Angeles (G07-02022) and Stellenbosch University Institutional Review Board (N08/08/218). This study was approved by the University of Cape Town (HREC REF 835/2015). 


\section{Results}

A total of 594 pregnant women were recruited from 12 control arm neighbourhoods, each comprising 20-60 participants. After excluding late-entry participants $(n=94)$, and participants who gave birth to twins $(n=2)$, who died or whose baby died during the study (from baseline to 18-month postpartum; $n=52$ ), the final sample was 446 (Online Resource 1). Participants excluded from the analyses were recruited earlier in pregnancy (median, $\mathrm{Mdn}$ 17.5 weeks, interquartile range, IQR 13-26) and reported higher EPDS scores at baseline (Mdn 13, IQR 7-19), compared to those included (gestation: Mdn 27.5 weeks, IQR 21-34, $p<0.001$; EPDS: Mdn 10, IQR 5-16, $p=0.011$ ).

Baseline characteristics of the final sample are presented in Table 1. Participants had a mean age of 26 years (standard deviation, SD 5.30), and were recruited, on average, at 26.6-week gestation (SD 8.03), corresponding to the 6th month of gestation. Only a minority reported completing high school $(n=110,24.7 \%)$, working $(n=83$, $18.6 \%)$, and not having a partner $(n=27,6.1 \%)$. Over a third $(n=174,39.0 \%)$ reported having experienced IPV in the previous year and $26.2 \%(n=107)$ reported being HIV-positive at baseline.

Compared to participants who were followed-up, participants lost to follow-up $(n=38,8.5 \%)$ reported lower baseline EPDS scores (Mdn 6, IQR 2-12 vs. Mdn 10, IQR 5-16, $U=2.79, p=0.005)$ and the majority were from the lower wealth category $\left(n=26,68.4 \%\right.$ vs. $n=197,48.3 \%, \chi^{2}=5.64$, $p=0.026$ ). At 36-month postpartum, a greater proportion of participants lost to follow-up $(n=73,16.4 \%)$ were from the lower wealth category ( $n=46,63.0$ vs. $n=177,47.5 \%$, $\left.\chi^{2}=5.91, p=0.021\right)$, and a smaller proportion reported experiencing IPV $\left(n=21,28.8 \%\right.$ vs. $n=153,41.0 \%, \chi^{2}=3.85$, $p=0.050)$.

\section{Trajectories}

The LCGA indices led to a four-class model (Table 2), which had the highest entropy (0.963) and fit significantly better than a three-class model, according to the LMRT and BLRT values (Table 2). The smallest class included $4.5 \%$ of the sample $(n=20)$. The change in mean EPDS scores over time among women in each of the four classes (Fig. 4.2) suggests a combination of chronic and transient symptom trajectories: (1) chronic low trajectory $(n=317,71.1 \%)$,
Table 1 Characteristics of participants at baseline, and across allocated trajectory

\begin{tabular}{|c|c|c|c|c|c|c|c|c|c|c|}
\hline \multirow[t]{2}{*}{ Variable } & \multicolumn{2}{|c|}{$\begin{array}{l}\text { Total } \\
(n=446)\end{array}$} & \multicolumn{2}{|c|}{$\begin{array}{l}\text { Chronic } \\
\text { low } \\
(n=317)\end{array}$} & \multicolumn{2}{|c|}{$\begin{array}{l}\text { Late } \\
\text { postpartum } \\
(n=45)\end{array}$} & \multicolumn{2}{|c|}{$\begin{array}{l}\text { Early } \\
\text { postpartum } \\
(n=64)\end{array}$} & \multicolumn{2}{|c|}{$\begin{array}{l}\text { Chronic } \\
\text { high } \\
(n=20)\end{array}$} \\
\hline & $n$ & $\%$ & $n$ & $\%$ & $n$ & $\%$ & $n$ & $\%$ & $n$ & $\%$ \\
\hline \multicolumn{11}{|l|}{ Socio-demographic characteristics } \\
\hline Age (mean, SD) & 26.0 & 5.30 & 26.0 & 5.23 & 26.7 & 5.69 & 25.2 & 5.31 & 27.1 & 5.58 \\
\hline Did not complete high school & 336 & 75.3 & 232 & 73.2 & 32 & 71.1 & 54 & 84.4 & 18 & 90.0 \\
\hline No current partner & 27 & 6.1 & 17 & 5.4 & 1 & 2.2 & 7 & 10.9 & 2 & 10.0 \\
\hline Currently unemployment & 363 & 81.4 & 246 & 77.6 & 35 & 77.8 & 62 & 96.9 & 20 & 100.0 \\
\hline Lower wealth & 223 & 50.0 & 160 & 50.5 & 21 & 46.7 & 32 & 50.0 & 10 & 50.0 \\
\hline Not Living with father of child & 209 & 46.9 & 148 & 46.7 & 22 & 48.9 & 30 & 46.9 & 9 & 45.0 \\
\hline Experienced IPV in past year & 174 & 39.0 & 112 & 35.3 & 23 & 51.1 & 30 & 46.9 & 9 & 45.0 \\
\hline Low social support $(\text { score } \leq \text { median })^{a}$ & 227 & 50.9 & 148 & 46.7 & 27 & 60.0 & 37 & 57.8 & 15 & 75.0 \\
\hline \multicolumn{11}{|l|}{ Obstetric characteristics } \\
\hline Gestation (in weeks) (mean, SD) & 26.6 & 8.03 & 26.6 & 8.02 & 26.1 & 8.66 & 27.0 & 7.31 & 23.9 & 8.38 \\
\hline Primigravida & 155 & 34.8 & 107 & 33.8 & 19 & 42.2 & 24 & 37.5 & 5 & 25.0 \\
\hline Primipara & 173 & 38.8 & 119 & 37.5 & 21 & 46.7 & 28 & 43.8 & 5 & 25.0 \\
\hline Unplanned pregnancy & 322 & 72.4 & 224 & 70.9 & 37 & 82.2 & 46 & 71.9 & 15 & 75.0 \\
\hline Unwanted pregnancy & 25 & 5.6 & 14 & 4.4 & 2 & 4.4 & 6 & 9.4 & 3 & 15.0 \\
\hline Previous miscarriage $(n=328)$ & 18 & 6.2 & 12 & 5.7 & 2 & 7.7 & 4 & 10.0 & 0 & 0 \\
\hline \multicolumn{11}{|l|}{ Health characteristics } \\
\hline EPDS score (mean, SD) & 10.9 & 6.86 & 9.9 & 6.54 & 12.2 & 6.51 & 13.1 & 7.50 & 15.7 & 6.96 \\
\hline Risky drinking $^{\mathrm{b}}$ & 20 & 4.5 & 14 & 4.4 & 1 & 2.2 & 2 & 3.2 & 3 & 15.0 \\
\hline HIV-positive status $(n=411)$ & 107 & 26.2 & 65 & 22.3 & 13 & 32.5 & 22 & 37.9 & 7 & 36.8 \\
\hline
\end{tabular}

${ }^{\mathrm{a}}$ Calculated as number of friends/relatives multiplied by frequency of contact

${ }^{b}$ Defined as a score of three or more on AUDIT-C 
Table 2 Latent class growth analysis: comparison of models with different numbers of classes

\begin{tabular}{lllllll}
\hline Classes & AIC & BIC & Entropy & $\begin{array}{l}\text { Size }(\%) \text { of small- } \\
\text { est class }\end{array}$ & LMRT statistic $(p$ value $)$ & BLRT statistic $(p$ value $)$ \\
\hline 2 & 11155.037 & 11200.140 & 0.955 & 15.0 & $559.562(<0.001)$ & $-5857.765(<0.001)$ \\
3 & 10956.411 & 11017.916 & 0.951 & 14.1 & $198.491(0.012)$ & $-5566.518(0.011)$ \\
4 & 10735.106 & 10813.012 & 0.963 & 4.5 & $220.278(<0.001)$ & $-5463.206(<0.001)$ \\
5 & 10686.188 & 10780.495 & 0.851 & 4.2 & $54.677(0.531)$ & $-5348.553(0.517)$ \\
6 & 10634.828 & 10745.536 & 0.875 & 3.8 & $57.023(0.188)$ & $-5320.094(0.180)$ \\
7 & 10618.594 & 10745.704 & 0.866 & 3.8 & $23.280(0.553)$ & $-5290.414(0.546)$ \\
\hline
\end{tabular}

AIC Akaike Information Criterion, BIC Bayesian Information Criterion, BLRT Bootstrap Likelihood Ratio Test, LMRT Lo-Mendell-Rubin Test

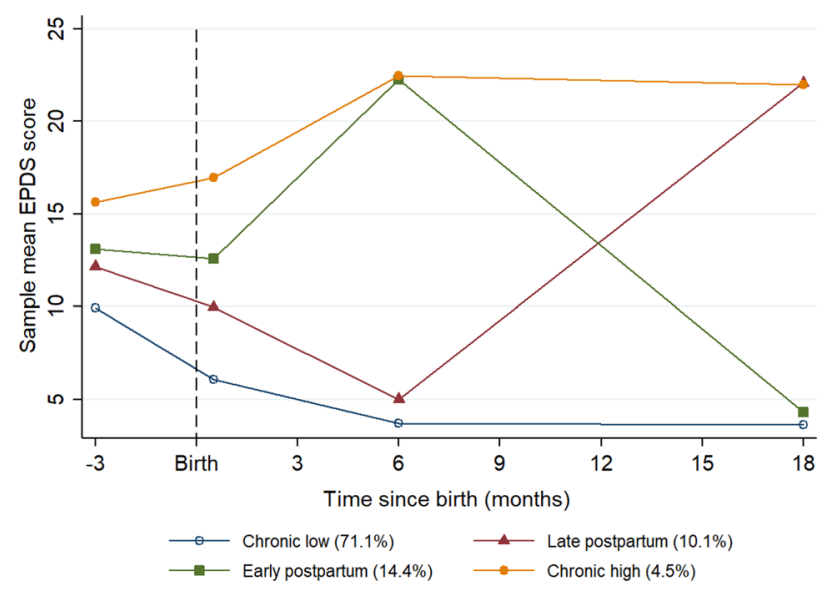

Fig. 1 Sample mean EPDS curves of the LCGA four-class solution

with a highest mean EPDS score at baseline (mean 9.9, SD 6.54), which steadily decreases; (2) late postpartum trajectory $(n=45,10.1 \%)$, with a slightly higher baseline mean score which increases sharply to 22.1 (SD 1.95) at 18-month postpartum; (3) early postpartum trajectory $(n=65,14.4 \%)$, with a mean baseline scores just above the severity cutoff of 13 [70], which reaches a maximum mean score of 22.3 (SD 3.6) at 6-month postpartum and declines to very low levels at 18-month postpartum; and (4) chronic high trajectory $(n=20,4.5 \%)$, with a mean baseline score of 15.7 (SD 6.96), which increases steadily to 22.5 (SD 3.79) at 6 months and stabilises (Fig. 1).

\section{Predictors}

The results of the univariate multinomial logistic regressions to assess baseline predictors of trajectories are presented in Table 3. The chronic low trajectory was used as the reference category. The risk of belonging to the chronic high trajectory, vs. the chronic low trajectory, among those who reported below average social support, an unwanted pregnancy and risky drinking was 3.43 (95\% CI 1.22, 9.65), 3.82 (95\% CI 1.00, 14.57) and 3.82 (95\% CI 1.00,
14.57) times as high, respectively, as the risk of belonging to this trajectory when these characteristics were not reported. The risk of belonging to the early postpartum trajectory among those who were unemployed and those who were HIV-positive was 8.95 (95\% CI 2.14, 37.48) and $2.13(95 \%$ CI $1.17,3.88)$ times as high as the risk of belonging to this trajectory when participants reported being employed and HIV negative, respectively. The risk of belonging to the late postpartum trajectory among those who reported IPV in the year preceding recruitment was $1.91(95 \%$ CI 1.02, 3.59) times as high as the risk of belonging to this trajectory when no IPV was reported.

\section{Child outcomes}

The results of the linear and logistic regressions assessing child outcomes at 18 and 36 months in relation with the trajectories, adjusted for age, education and wealth, are presented in Table 4. Again, the chronic low trajectory was used as the reference trajectory.

\section{8-month outcomes}

Children of participants allocated to the late postpartum trajectory had significantly lower weight-to-length (mean 0.11, SD 1.36) and weight-for-age $z$-scores (mean -0.22 , SD 1.09) compared to children of participants in the chronic low trajectory (weight-to-length $z$-score: mean 0.91 , SD 1.33; adjusted $\beta(\mathrm{a} \beta)-0.81 ; 95 \% \mathrm{CI}-1.27$, -0.34 ; weight-for-age $z$-scores: mean 0.41 , SD 1.22 , a $\beta$ $-0.63 ; 95 \% \mathrm{CI}-1.05,-0.21)$. The same pattern could be seen among children of participants allocated to the early postpartum trajectory, with children reporting lower weight-to-length (mean 0.36 , SD 1.33 , a $\beta-0.52 ; 95 \%$ CI $-0.92,-0.11$ ) and weight-to-age $z$-scores (mean -0.09 , SD $1.23 ; \mathrm{a} \beta-0.45 ; 95 \% \mathrm{CI}-0.81,-0.09)$ compared to children of participants in the chronic low trajectory. 
Table 3 Baseline predictors of classes identified through the latent class growth analysis, in comparison with chronic low trajectory

\begin{tabular}{|c|c|c|c|c|c|c|c|c|c|}
\hline \multirow[t]{2}{*}{ Variable } & \multicolumn{3}{|c|}{ Late postpartum $(n=45)$} & \multicolumn{3}{|c|}{ Early postpartum $(n=64)$} & \multicolumn{3}{|c|}{ Chronic high $(n=20)$} \\
\hline & RRR & $95 \% \mathrm{CI}$ & $p$ & RRR & $95 \% \mathrm{CI}$ & $p$ & RRR & $95 \% \mathrm{CI}$ & $p$ \\
\hline \multicolumn{10}{|l|}{ Socio-demographics characteristics } \\
\hline Age & 1.03 & $0.97-1.09$ & 0.389 & 0.97 & $0.92-1.02$ & 0.274 & 1.04 & $0.96-1.13$ & 0.374 \\
\hline Did not complete high school & 0.90 & $0.45-1.80$ & 0.769 & 1.98 & $0.96-4.06$ & 0.063 & 3.30 & $0.75-14.51$ & 0.115 \\
\hline Currently unemployed & 1.01 & $0.48-2.14$ & 0.979 & 8.95 & $2.14-37.48$ & 0.003 & - & - & - \\
\hline Lower wealth & 1.08 & $0.79-1.48$ & 0.633 & 1.01 & $0.77-1.32$ & 0.945 & 1.01 & $0.64-1.59$ & 0.967 \\
\hline No partner & 0.40 & $0.05-3.08$ & 0.379 & 2.16 & $0.86-5.44$ & 0.103 & 1.95 & $0.42-9.12$ & 0.394 \\
\hline Not living with father of child & 1.09 & $0.58-2.04$ & 0.782 & 1.01 & $0.59-1.73$ & 0.978 & 0.93 & $0.38-2.32$ & 0.883 \\
\hline Lower social support (score $\leq$ median $)^{\mathrm{a}}$ & 1.71 & $0.91-3.24$ & 0.097 & 1.56 & $0.91-2.69$ & 0.106 & 3.43 & $1.22-9.65$ & 0.020 \\
\hline Experienced IPV in past year & 1.91 & $1.02-3.59$ & 0.043 & 1.62 & $0.94-2.78$ & 0.083 & 1.50 & $0.60-3.72$ & 0.385 \\
\hline \multicolumn{10}{|l|}{ Obstetric characteristics } \\
\hline Primigravida & 1.43 & $0.76-2.71$ & 0.266 & 1.18 & $0.67-2.06$ & 0.565 & 0.65 & $0.23-1.85$ & 0.423 \\
\hline Primipara & 1.46 & $0.78-2.73$ & 0.241 & 1.29 & $0.75-2.23$ & 0.353 & 0.55 & $0.20-1.56$ & 0.265 \\
\hline Unplanned pregnancy & 1.90 & $0.85-4.24$ & 0.117 & 1.05 & $0.58-1.91$ & 0.874 & 1.23 & $0.44-3.49$ & 0.694 \\
\hline Unwanted pregnancy & 1.01 & $0.22-4.58$ & 0.993 & 2.24 & $0.83-6.07$ & 0.113 & 3.82 & $1.00-14.57$ & 0.050 \\
\hline \multicolumn{10}{|l|}{ Health characteristics } \\
\hline Risky drinking $^{\mathrm{b}}$ & 0.49 & $0.06-3.83$ & 0.498 & 0.71 & $0.16-3.20$ & 0.655 & 3.82 & $1.00-14.57$ & 0.050 \\
\hline HIV positive & 1.68 & $0.82-3.44$ & 0.155 & 2.13 & $1.17-3.88$ & 0.013 & 2.04 & $0.77-5.39$ & 0.151 \\
\hline
\end{tabular}

$R R R$ relative risk ratios, $C I$ confidence intervals

${ }^{\mathrm{a}}$ Calculated as number of friends/relatives multiplied by frequency of contact

${ }^{b}$ Defined as a score of three or more on AUDIT-C

\section{6-month outcomes}

Results indicate that children of participants in the late postpartum trajectory had lower weight-for-age $z$-scores (mean -0.20, SD 0.99) compared to children of participants allocated to the chronic low trajectory (mean 0.23 , SD 1.09; $\mathrm{a} \beta-0.43 ; 95 \% \mathrm{CI}-0.78,-0.07)$. Children of participants in the early postpartum trajectory reported significantly lower length-for-age (mean -1.83 , SD 1.07, a $\beta-0.55 ; 95 \%$ CI $-0.90,-0.21)$ and weight-for-age $z$-scores (mean -0.34 , SD 0.94; $\beta-0.54 ; 95 \%$ CI -0.87 , $-0.21)$.

Scores on the executive functioning battery did not differ across trajectories. There were also no differences in internalising, externalising or total problem scores on the CBCL, nor differences in total difficulty score on the SDQ. However, children of participants in the chronic high trajectory reported greater emotional symptom scores (mean 2.2, SD 2.66), and children of participants in the late postpartum trajectory had greater peer problem scores (mean 3.0, SD 1.26), compared to children in the chronic low trajectory (emotional: mean 1.1, SD 1.47, a $\beta$ 0.96; 95\% CI 0.17, 1.75; peer problems: mean 2.6, SD 1.13, a $\beta 0.42$ 95\% CI 0.0.02, $0.81)$. Finally, children of participants in the early postpartum trajectory (mean 8.2, SD $1.62 ; \mathrm{a} \beta 0.85 ; 95 \%$ CI 0.21 , 1.50 ) and in the chronic high trajectory (mean 8.5, SD 1.5; a $\beta 1.19 ; 95 \%$ CI $0.14,2.24)$ reported greater SDQ prosocial scores compared to children of participants in the chronic low trajectory (mean 7.5, SD 2.22).

\section{Discussion}

This study sought to identify latent trajectories of depressive symptoms from pregnancy to 18-month postpartum, and their predictors, among low-income perinatal women in South Africa. Altogether, the four trajectories identified support previous findings using similar modelling techniques $[30,33]$. First, the chronic low trajectory identified was reported in a previous study conducted in Africa [32], suggesting that, despite the high number and ongoing stressors experienced in LMICs, the majority of women remain at low risk of developing depressive symptoms during the perinatal period. Second, the early postpartum and late postpartum trajectories identified are also similar to those reported by Barthel et al. [32]. In their study conducted among perinatal women in Ghana and Côte d'Ivoire, they report that besides family and financial stress, none of the socio-demographic or psychosocial factors were associated with these trajectories. Findings were similar in our study: only IPV in the year leading to pregnancy was identified as a risk factor for late postpartum depressive symptoms, and unemployment and HIV-positive status as risk factors for early postpartum depressive symptoms. The latter finding corroborates past 


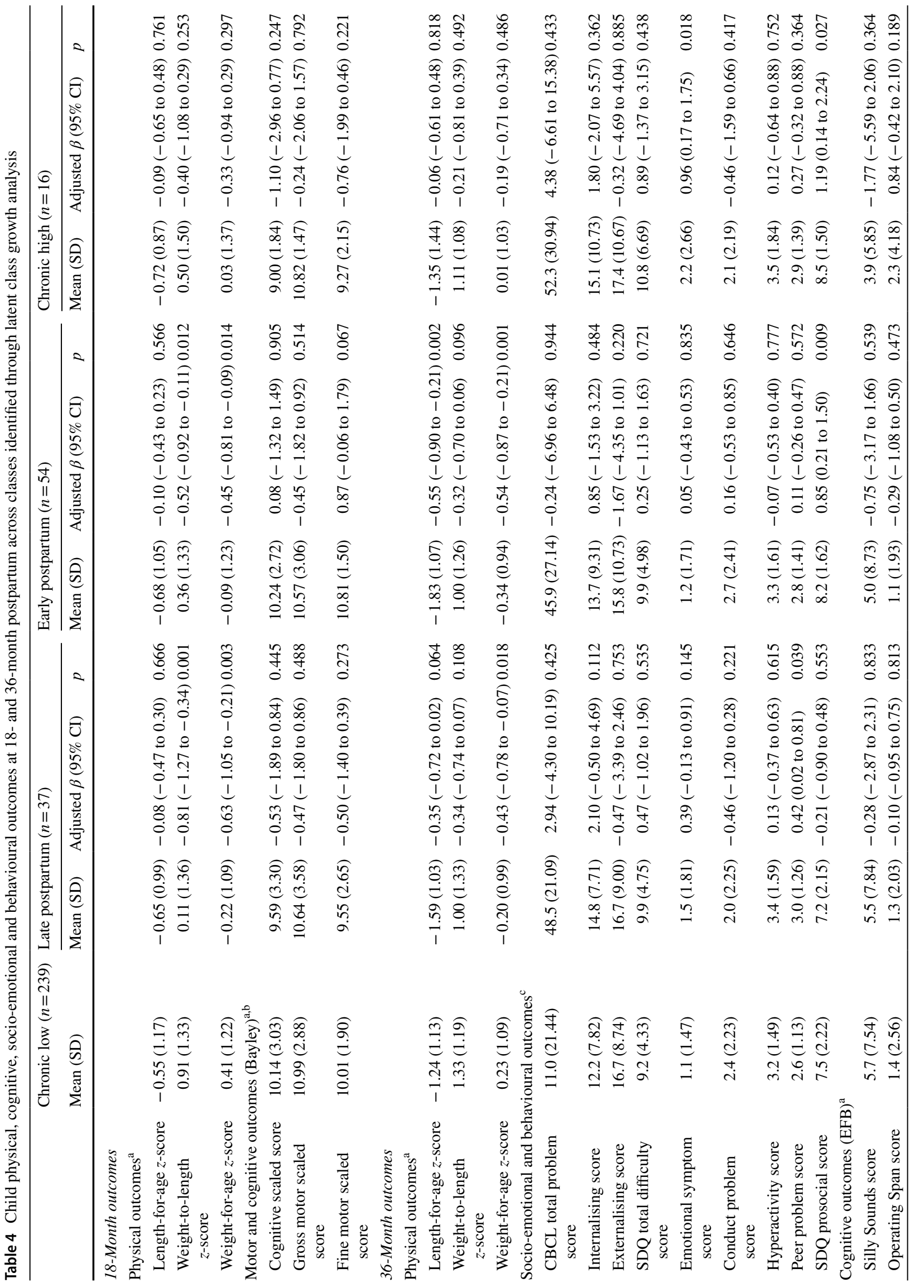


research in South Africa indicating that being diagnosed with HIV during pregnancy can put mothers at increased risk of postnatal depression [71]. The practical implications of inadequate resources to provide for a new baby, once the baby is born, could also explain the association found between unemployment and the early postpartum trajectory [72]. Finally, women with chronically severe symptoms, reported in the previous research in South Africa [31], seem to be a high-risk group, as they reported having less social support, were more likely to report an unwanted pregnancy and more likely to have been engaging in risky drinking behaviour during pregnancy. Likewise, the high-risk antenatal and postnatal trajectory in Baron et al. [31]'s study was associated with lower social support and heavy drinking during pregnancy.

The fact that two of the four trajectories identified have transient patterns indicate that a single screening for depressive symptoms may not be enough to identify women at risk of perinatal depression. Instead, repeated screening conducted throughout pregnancy and the first year postpartum would be more effective, perhaps in the context of antenatal and well-baby visits. Alternatively, and as our findings suggest, screening for other factors, such as lack of social support, alcohol use and IPV, in antenatal settings may be a way of identifying pregnant women who may be at risk of chronic symptoms or of worse symptoms later in the postpartum period.

The second aim of our study was to investigate the association between trajectories of perinatal depression symptoms and child outcomes at 18- and 36-month postpartum. Important distinctions were found in children's physical outcomes across trajectories, and this even after controlling for known demographic confounders, including wealth, suggesting that poorer physical outcomes were not solely due to children's social or economic environment. In addition, the fact that weight-to-length $z$-scores, a short-term response to inappropriate nutrition [15], and length-for-age $z$-scores, a longer term cumulative response to poor diet and recurrent illness [15], were lower at 18 and 36 months, respectively, among children of mothers in the early postpartum trajectory suggest that the effect of postnatal depressive symptoms may have long-lasting effects on child physical outcomes. Emerging evidence suggests that psychosocial interventions for depression which include parenting or caregiving components are the most effective in improving both maternal mental health and child outcomes [15]. Given the impact of depressive symptoms on children's physical outcomes in this study, it may be worth including a nutritional advice component in psychosocial interventions addressing perinatal depressive symptoms.

Surprisingly, the motor or cognitive outcomes of infants at 18 months, and executive functioning at 36 months, thought to be an indicator of learning disability even when 
cognitive abilities seem intact [73], did not differ across depressive symptom trajectories. There were also no differences in overall socio-emotional or behavioural outcomes on the SDQ or CBCL questionnaires at 36-month postpartum. However, the investigation of the SDQ subscales indicated that children of mothers with chronically high symptoms had greater emotional symptom problems, and children of mothers among the late postpartum trajectory had greater peer-related problems, compared to children of mothers with low depressive symptoms. This corroborates Vänskä et al. (2011)'s study, where no differences in externalising symptoms could be found across different trajectories of depression, but children of mothers with chronically high symptoms showed greater internalising symptoms compared to children of mothers with stable low symptoms.

As suggested by Prenoveau et al. [28], it may be that mothers' low positive affect may be more detrimental to children' emotional negativity specifically. However, the previous studies using similar modelling techniques did find that children of mothers with chronic or transient depressive symptoms reported poorer executive functioning and greater emotional and behavioural problems [71-76]. These studies were conducted in HICs, however, and the impact of depressive symptoms on children's development may differ in LMICs, where social and psychological factors, such as poverty and cultural norms, are likely to play an important role. It was beyond the scope of this study to investigate moderating or mediating factors of child development; however, future research in LMICs should to further our understanding of the underlying mechanisms between maternal depression and child development.

\section{Limitations}

Several limitations should be noted. First, while LCGA overcomes some of past research's pitfalls, assessments were still limited and wide apart. Our interpretation of 'chronic' symptoms, therefore, remains simplistic. With only one assessment conducted during pregnancy, we cannot assess whether symptoms fluctuated during pregnancy, or whether they were a continuation of pre-pregnancy symptoms. In addition, the second assessment was conducted within the first 2-week postpartum, so depressive symptoms may have reflected baby blues, rather than postnatal depression per se. Moreover, the EPDS is a valid measure of depressive symptoms during the perinatal period, but the exclusion of somatic symptoms means that it may not be valid at 18 or 36-month postpartum. The potential underestimation of depressive symptom severity at these assessments may have affected the 18-month mark of trajectories identified through LCGA. It may also have led to underestimate the effect of depressive symptoms on mothers' reports of child development at 36 months, which was controlled for in the analysis. This may partly explain the lack of association between depressive symptoms trajectories and behavioural outcomes.

A further limitation relating to the use of LCGA is that it assumes no intra-class variance in the growth parameters, which may have been too restrictive. However, allowing free variance for the intercepts produced a negligible improvement in fit and no substantive changes in the shape of the trajectories or in the overall distribution of the sample across classes. Models also did not converge when free variance was allowed for the slope and quadratic terms (i.e., moving from LCGA to growth mixture modelling). While this could be due to an insufficient sample size, this may reflect the absence of significant variability, suggesting that LCGA may be an appropriate method to model our data nonetheless.

The little intra-class variability also supports the fourclass model identified, despite the small sample size of the chronic high trajectory. However, a small sample size meant that we may not have had enough power to identify meaningful differences between trajectories, especially between the chronic high and low trajectories. A small sample size also meant that univariate, rather than multivariate, analyses of risk factors were more appropriate. However, though not reported here, the patterns of risk for each trajectory did not change substantially when multivariate analyses of risk factors were conducted despite the presence of broad confidence intervals.

Finally, all analyses conducted in Stata to assess predictors and child outcomes considered class membership as observed, rather than predicted by the LCGA model. However, given the high classification accuracy showed by our four-class model, it is unlikely that taking into account this uncertainty in regression models would have produced substantive changes in the interpretation of the results.

\section{Conclusions}

To our knowledge, this is the first study in Sub-Saharan Africa to have used GCMM to assess the course of perinatal depressive symptoms in relation with child development. Despite several limitations, our study has clear implications for the identification of at-risk populations and for the development of preventive interventions to promote maternal mental health and child development. Mothers remain at risk for developing severe symptoms after 1-year postpartum, and while several psychosocial factors can help identify pregnant women likely to suffer from chronic or late postpartum depression, policy makers and practitioners must recognise the need for multiple screening assessments throughout pregnancy and the first year postpartum. 
Our study does not support the idea that there are sensitive periods in which maternal depressive symptoms have qualitatively different impact on children's development. Instead, it seems the presence of depressive symptoms at any point during the perinatal period can have adverse effects on children's physical and socio-emotional outcomes. Further investigation in LMICs is warranted, with bigger samples and more frequent assessments. This will help tease out the relative importance of chronicity of symptoms vs. severity, identify whether antenatal and postnatal depressive symptoms have independent or interactive effects on child outcomes, and work towards identifying pathways between perinatal depression and child outcomes that are specific to LMICs.

Acknowledgements We would like to acknowledge Dr. Sujit Rathod, who provided insights into post-hoc analyses, and Ellen Almirol for providing support with access to the study materials and datasets. This study is an output of the PRogramme for Improving Mental health carE (PRIME). This work was supported by the UK Department for International Development (201446). The views expressed do not necessarily reflect the UK Government's official policies.

\section{Compliance with ethical standards}

Conflict of interest The authors declare that they have no conflict of interest.

Ethical standards Informed consent was given by all participants before their inclusion in the study. Personal details of participants were omitted from the data. The original cluster randomised controlled trial was approved by the Institutional Review Boards of the UCLA (G07-02-022) and Stellenbosch University Institutional Review Board (N08/08/218). This study was approved by the University of Cape Town (HREC REF 835/2015).

Open Access This article is distributed under the terms of the Creative Commons Attribution 4.0 International License (http://creativeco mmons.org/licenses/by/4.0/), which permits unrestricted use, distribution, and reproduction in any medium, provided you give appropriate credit to the original author(s) and the source, provide a link to the Creative Commons license, and indicate if changes were made.

\section{References}

1. Woody C, Ferrari A, Siskind D, Whiteford H, Harris M (2017) A systematic review and meta-regression of the prevalence and incidence of perinatal depression. J Affect Disord 219:86-92

2. van Heyningen T, Myer L, Onah M, Tomlinson M, Field S, Honikman S (2016) Antenatal depression and adversity in urban South Africa. J Affect Disord 203:121-129

3. Rochat TJ (2011) Depression among pregnant women testing for HIV in rural South Africa. Stellenbosch University, Stellenbosch

4. Manikkam L, Burns JK (2012) Antenatal depression and its risk factors: an urban prevalence study in KwaZulu-Natal. S Afr Med J 102(12):940-944

5. Hartley M, Tomlinson M, Greco E, Comulada WS, Stewart J, Le Roux I, Mbewu N, Rotheram-Borus MJ (2011) Depressed mood in pregnancy: prevalence and correlates in two Cape Town periurban settlements. Reprod Health 8(9):4755-4758

6. Redinger S, Norris S, Pearson R, Richter L, Rochat T (2017) First trimester antenatal depression and anxiety: prevalence and associated factors in an urban population in Soweto, South Africa. J Dev Orig Health Dis 9(1):30-40

7. Brittain K, Myer L, Koen N, Koopowitz S, Donald KA, Barnett W, Zar HJ, Stein DJ (2015) Risk factors for antenatal depression and associations with infant birth outcomes: results from a South African birth cohort study. Paediatr Perinat Epidemiol 29(6):505-514

8. Cooper PJ, Tomlinson M, Swartz L, Woolgar M, Murray L, Molteno C (1999) Post-partum depression and the mother-infant relationship in a South African peri-urban settlement. Br J Psychiatry 175(6):554-558

9. Verkuijl NE, Richter L, Norris SA, Stein A, Avan B, Ramchandani PG (2014) Postnatal depressive symptoms and child psychological development at 10 years: A prospective study of longitudinal data from the South African Birth to Twenty cohort. Lancet Psychiatry 1(6):454-460. https://doi.org/10.1016/S2215-0366(14)70361-X

10. Ramchandani PG, Richter LM, Stein A, Norris SA (2009) Predictors of postnatal depression in an urban South African cohort. J Affect Disord 113(3):279-284

11. Goodman SH, Rouse MH, Connell AM, Broth MR, Hall CM, Heyward D (2011) Maternal depression and child psychopathology: a meta-analytic review. Clin Child Fam Psychol Rev 14(1):1-27

12. Kingston D, McDonald S, Austin M-P, Tough S (2015) Association between prenatal and postnatal psychological distress and toddler cognitive development: a systematic review. PLoS One 10(5): 0126929

13. Liu Y, Kaaya S, Chai J, McCoy D, Surkan P, Black M, Sutter-Dallay A-L, Verdoux H, Smith-Fawzi M (2017) Maternal depressive symptoms and early childhood cognitive development: a metaanalysis. Psychol Med 47(4):680-689

14. Kingston D, Tough S, Whitfield H (2012) Prenatal and postpartum maternal psychological distress and infant development: a systematic review. Child Psychiatry Hum Dev 43(5):683-714

15. Parsons CE, Young KS, Rochat TJ, Kringelbach M, Stein A (2012) Postnatal depression and its effects on child development: a review of evidence from low-and middle-income countries. $\mathrm{Br}$ Med Bull 101(1):57-59

16. Walker CLF, Lamberti L, Adair L, Guerrant RL, Lescano AG, Martorell R, Pinkerton RC, Black RE (2012) Does childhood diarrhea influence cognition beyond the diarrhea-stunting pathway? PLoS One 7(10):e47908

17. Black RE, Cousens S, Johnson HL, Lawn JE, Rudan I, Bassani DG, Jha P, Campbell H, Walker CF, Cibulskis R (2010) Global, regional, and national causes of child mortality in 2008: a systematic analysis. Lancet 375(9730):1969-1987

18. Gelaye B, Rondon MB, Araya R, Williams MA (2016) Epidemiology of maternal depression, risk factors, and child outcomes in low-income and middle-income countries. Lancet Psychiatry 3(10):973-982

19. Surkan PJ, Patel SA, Rahman A (2016) Preventing infant and child morbidity and mortality due to maternal depression. Best Pract Res Clin Obstet Gynaecol 36:156-168

20. Avan B, Richter LM, Ramchandani PG, Norris SA, Stein A (2010) Maternal postnatal depression and children's growth and behaviour during the early years of life: exploring the interaction between physical and mental health. Arch Dis Child 95(9):690695. https://doi.org/10.1136/adc.2009.164848

21. Tomlinson M, Cooper P, Stein A, Swartz L, Molteno C (2006) Post-partum depression and infant growth in a South African periurban settlement. Child Care Health Dev 32(1):81-86 
22. Banti S, Mauri M, Oppo A, Borri C, Rambelli C, Ramacciotti D, Montagnani MS, Camilleri V, Cortopassi S, Rucci P (2011) From the third month of pregnancy to 1 year postpartum. Prevalence, incidence, recurrence, and new onset of depression. Results from the Perinatal Depression-Research \& Screening Unit study. Compr Psychiatry 52(4):343-351

23. Martini J, Petzoldt J, Einsle F, Beesdo-Baum K, Hofler M, Wittchen HU (2015) Risk factors and course patterns of anxiety and depressive disorders during pregnancy and after delivery: a prospective-longitudinal study. J Affect Disord 175:385-395. https://doi.org/10.1016/j.jad.2015.01.012

24. Woolhouse H, Gartland D, Mensah F, Giallo R, Brown S (2016) Maternal depression from pregnancy to 4 years postpartum and emotional/behavioural difficulties in children: results from a prospective pregnancy cohort study. Arch Women's Ment Health 19(1):141-151

25. Stein A, Pearson RM, Goodman SH, Rapa E, Rahman A, McCallum M, Howard LM, Pariante CM (2014) Effects of perinatal mental disorders on the fetus and child. Lancet 384(9956):1800-1819

26. Sohr-Preston SL, Scaramella LV (2006) Implications of timing of maternal depressive symptoms for early cognitive and language development. Clin Child Fam Psychol Rev 9(1):65-83

27. Rotheram-Fuller EJ, Tomlinson M, Scheffler A, Weichle TW, Hayati Rezvan P, Comulada WS, Rotheram-Borus MJ (2018) Maternal patterns of antenatal and postnatal depressed mood and the impact on child health at 3-years postpartum. J Consult Clin Psychol 86(3):218

28. Prenoveau JM, Craske MG, West V, Giannakakis A, Zioga M, Lehtonen A, Davies B, Netsi E, Cardy J, Cooper P (2017) Maternal postnatal depression and anxiety and their association with child emotional negativity and behavior problems at two years. Dev Psychol 53(1):50

29. Pettit J, Lewinsohn P, Roberts R, Seeley J, Monteith L (2009) The long-term course of depression: development of an empirical index and identification of early adult outcomes. Psychol Med 39(3):403-412

30. Baron E, Bass J, Murray SM, Schneider M, Lund C (2017) A systematic review of growth curve mixture modelling literature investigating trajectories of perinatal depressive symptoms and associated risk factors. J Affect Disord 223:194-208

31. Garman E, Schneider M, Lund C (2019) Perinatal depressive symptoms among low-income South Africanwomen at risk of depression: trajectories and predictors. BMC Women's Health (Under review)

32. Barthel D, Kriston L, Fordjour D, Mohammed Y, Kra-Yao ED, Kotchi CEB, Armel EJK, Eberhardt KA, Feldt T, Hinz R (2017) Trajectories of maternal ante-and postpartum depressive symptoms and their association with child-and mother-related characteristics in a West African birth cohort study. PLoS One 12(11):e0187267

33. Santos H, Tan X, Salomon R (2017) Heterogeneity in perinatal depression: how far have we come? A systematic review. Arch Women's Ment Health 20(1):1-13

34. Herba CM, Glover V, Ramchandani PG, Rondon MB (2016) Maternal depression and mental health in early childhood: an examination of underlying mechanisms in low-income and middle-income countries. Lancet Psychiatry 3(10):983-992

35. Rotheram-Borus MJ, Le Roux IM, Tomlinson M, Mbewu N, Comulada WS, Le Roux K, Stewart J, O'Connor MJ, Hartley M, Desmond K (2011) Philani Plus (+): a Mentor Mother community health worker home visiting program to improve maternal and infants' outcomes. Prev Sci 12(4):372-388

36. le Roux IM, Tomlinson M, Harwood JM, O'CONNOR MJ, Worthman CM, Mbewu N, Stewart J, Hartley M, Swendeman D, Comulada WS (2013) Outcomes of home visits for pregnant mothers and their infants: a cluster randomised controlled trial. AIDS (London England) 27(9):1461

37. Rotheram-Borus MJ, Richter LM, Van Heerden A, Van Rooyen H, Tomlinson M, Harwood JM, Comulada WS, Stein A (2014) A cluster randomized controlled trial evaluating the efficacy of peer mentors to support South African women living with HIV and their infants. PLoS One. https://doi.org/10.1371/journ al.pone. 0084867

38. Tomlinson M, Rotheram-Borus MJ, Harwood J, Le Roux IM, O'Connor M, Worthman C (2015) Community health workers can improve child growth of antenatally-depressed, South African mothers: a cluster randomized controlled trial. BMC Psychiatry 15(1):225

39. Tomlinson M, Rotheram-Borus MJ, Le Roux IM, Youssef M, Nelson SH, Scheffler A, Weiss RE, O'Connor M, Worthman CM (2016) Thirty-six-month outcomes of a generalist paraprofessional perinatal home visiting intervention in South Africa on maternal health and child health and development. Prev Sci 17(8):937-948

40. Brislin R (1986) The wording and translation of research instruments. In: Lonner W, Berry J (eds) Field methods in cross-cultural research. Sage, London

41. Booysen F, Van Der Berg S, Burger R, Von Maltitz M, Du Rand $\mathrm{G}$ (2008) Using an asset index to assess trends in poverty in seven Sub-Saharan African countries. World Dev 36(6):1113-1130

42. Cox JL, Holden JM, Sagovsky R (1987) Detection of postnatal depression. Development of the 10-item Edinburgh Postnatal Depression Scale. Br J Psychiatry 150(6):782-786

43. Cox JL, Holden J (1994) Perinatal psychiatry: use and misuse of the Edinburgh Postnatal Depression Scale. Gaskell

44. Lawrie T, Hofmeyr G, De Jager M, Berk M (1998) Validation of the Edinburgh Postnatal Depression Scale on a cohort of South African women. S Afr Med J 88 (10):1340-1344

45. De Bruin GP, Swartz L, Tomlinson M, Cooper PJ, Molteno C (2004) The factor structure of the Edinburgh Postnatal Depression scale in a South African peri-urban settlement. S Afr J Psychol 34(1):113-121

46. Dawson DA, Grant BF, Stinson FS (2005) The AUDIT-C: screening for alcohol use disorders and risk drinking in the presence of other psychiatric disorders. Compr Psychiatry 46(6):405-416

47. Saunders JB, Aasland OG, Babor TF, De la Fuente JR, Grant M (1993) Development of the alcohol use disorders identification test (AUDIT). WHO collaborative project on early detection of persons with harmful alcohol consumption-II. Addict Abingdon 88:791-791

48. Parry CDH (2001) Alcohol and other drug abuse. In: Ntuli A, Crisp N, Clarke E, Barron P (eds) South African health review: 2000. Health Systems Trust, Durban, pp 441-451

49. Kalichman SC, Simbayi LC, Vermaak R, Cain D, Smith G, Mthebu J, Jooste S (2008) Randomized trial of a communitybased alcohol-related HIV risk-reduction intervention for men and women in Cape Town South Africa. Ann Behav Med 36(3):270-279

50. WHO Multicentre Growth Reference Study Group (2006) WHO child growth standards: length/height-for-age, weight-for-age, weight-for-length, weight-for-height and body mass index-for-age: methods and development. World Health Organization, Geneva

51. Bayley N (2003) Bayley scales of infant development, 2nd edn. Psychological Corporation, San Antonio

52. Blair C, Zelazo PD, Greenberg MT (2016) Measurement of executive function in early childhood: a special issue of developmental neuropsychology. Psychology Press, New York

53. Achenbach TM, Rescorla LA (2000) Manual for the ASEBA preschool forms and profiles. University of Vermont, Research Center for Children, Youth, \& Families, Burlington

54. Goodman R (1997) The Strengths and Difficulties Questionnaire: a research note. J Child Psychol Psychiatry 38(5):581-586 
55. Cluver L, Gardner F (2006) The psychological well-being of children orphaned by AIDS in Cape Town, South Africa. Ann Gen Psychiatry 5(1):8

56. Muthén L, Muthén B (1998-2015) Mplus user's guide, 7th edn. Muthén \& Muthén, Los Angeles

57. StataCorp (2015) Stata statistical software: release 14. StataCorp LP, College Station

58. Berlin KS, Parra GR, Williams NA (2014) An introduction to latent variable mixture modeling (part 2): longitudinal latent class growth analysis and growth mixture models. J Pediatr Psychol 39(2):188-203

59. Froot KA (1989) Consistent covariance matrix estimation with cross-sectional dependence and heteroskedasticity in financial data. J Finan Quant Anal 24(3):333-355

60. Lt Hu, Bentler PM (1999) Cutoff criteria for fit indexes in covariance structure analysis: conventional criteria versus new alternatives. Struct Equ Model Multidiscip J 6(1):1-55

61. Raftery AE (1995) Bayesian model selection in social research. Sociol Methodol 25:111-164

62. Akaike H (1983) Information measures and model selection. Bull Int Stat Inst 50(1):277-291

63. Ramaswamy V, DeSarbo WS, Reibstein DJ, Robinson WT (1993) An empirical pooling approach for estimating marketing mix elasticities with PIMS data. Market Sci 12(1):103-124

64. Lo Y, Mendell NR, Rubin DB (2001) Testing the number of components in a normal mixture. Biometrika 88(3):767-778

65. McLachlan G, Peel D (2004) Finite mixture models. Wiley, New Jersey

66. Rahman A, Iqbal Z, Bunn J, Lovel H, Harrington R (2004) Impact of maternal depression on infant nutritional status and illness: a cohort study. Arch Gen Psychiatry 61(9):946-952. https://doi. org/10.1001/archpsyc.61.9.946

67. Rowe ML, Goldin-Meadow S (2009) Differences in early gesture explain SES disparities in child vocabulary size at school entry. Science 323(5916):951-953

68. Grote NK, Bridge JA, Gavin AR, Melville JL, Iyengar S, Katon WJ (2010) A meta-analysis of depression during pregnancy and the risk of preterm birth, low birth weight, and intrauterine growth restriction. Arch Gen Psychiatry 67(10):1012-1024

69. Müller JM, Achtergarde S, Furniss T (2011) The influence of maternal psychopathology on ratings of child psychiatric symptoms: an SEM analysis on cross-informant agreement. Eur Child Adolesc Psychiatry 20(5):241-252

70. Rochat TJ, Tomlinson M, Newell M-L, Stein A (2013) Detection of antenatal depression in rural HIV-affected populations with short and ultrashort versions of the Edinburgh Postnatal Depression Scale (EPDS). Arch Women's Ment Health 16(5):401-410

71. Rochat TJ, Richter LM, Doll HA, Buthelezi NP, Tomkins A, Stein A (2006) Depression among pregnant rural South African women undergoing HIV testing. JAMA 295(12):1373-1378

72. Lee AM, Lam SK, Lau SMSM, Chong CSY, Chui HW, Fong DYT (2007) Prevalence, course, and risk factors for antenatal anxiety and depression. Obstet Gynecol 110(5):1102-1112

73. Riggs NR, Blair CB, Greenberg MT (2004) Concurrent and 2-year longitudinal relations between executive function and the behavior of 1st and 2nd grade children. Child Neuropsychol 9(4):267-276

74. van der Waerden J, Galéra C, Larroque B, Saurel-Cubizolles M-J, Sutter-Dallay A-L, Melchior M (2015) Maternal depression trajectories and children's behavior at age 5 years. J Pediatr 166(6):1440-1448. https://doi.org/10.1016/j.jpeds.2015.03.002

75. Giallo R, Woolhouse H, Gartland D, Hiscock H, Brown S (2015) The emotional-behavioural functioning of children exposed to maternal depressive symptoms across pregnancy and early childhood: a prospective Australian pregnancy cohort study. Eur Child Adolesc Psychiatry 24(10):1233-1244. https://doi.org/10.1007/ s00787-014-0672-2

76. Vänskä M, Punamäki RL, Tolvanen A, Lindblom J, Flykt M, Unkila-Kallio L, Tiitinen A, Repokari L, Sinkkonen J, Tulppala M (2011) Maternal pre- and postnatal mental health trajectories and child mental health and development: prospective study in a normative and formerly infertile sample. Int J Behav Dev 35(6):517-531. https://doi.org/10.1177/0165025411417505 